\title{
Латентное накопление поверхностных состояний в МОП структурах после ионизирующего облучения
}

\author{
(C) О.В. Александров \\ Санкт-Петербургский государственный электротехнический университет „ЛЭТИ“, \\ 197376 Санкт-Петербург, Россия \\ E-mail: Aleksandr_ov@mail.ru
}

Поступила в Редакцию 1 марта 2021 г.

В окончательной редакции 12 марта 2021 г.

Принята к публикации 12 марта 2021 г.

\begin{abstract}
Разработана новая количественная модель латентного накопления поверхностных состояний в МОП структурах после ионизирующего облучения и длительного отжига. Модель базируется на образовании под воздействием ионизирующего облучения ионов водорода $\mathrm{H}^{+}$не только в тонком подзатворном диэлектрике, но и в прилегающем толстом полевом диэлектрике, и их последующем дисперсионном переносе к межфазной границе с кремниевой подложкой. Плотность латентных поверхностных состояний определяется уравнением баланса пассивации и депассивации $P_{b}$-центров на межфазной границе $\mathrm{SiO}_{2}-\mathrm{Si}$ ионами водорода. Модель позволяет объяснить сопутствующее падение объемного заряда, а также спад плотности поверхностных состояний после завершения роста и удовлетворительно описывает экспериментальные данные.
\end{abstract}

Ключевые слова: ионизирующее облучение, МОП структура, латентные поверхностные состояния, дисперсионный транспорт, моделирование.

DOI: 10.21883/FTP.2021.07.51016.9641

\section{1. Введение}

После ионизирующего облучения (ИО) структур металл-оксид-полупроводник (МОП структур) помимо нормального образования поверхностных состояний (ПС) на межфазной границе (МФГ) $\mathrm{Si}-\mathrm{SiO}_{2}$ наблюдается так называемое латентное накопление ПС, заключающееся в росте плотности ПС при длительных временах выдержки или отжига $\left(t>10^{5}-10^{6}\right.$ с при $\left.25^{\circ} \mathrm{C}\right)[1-5]$. Появление таких латентных ПС сопровождается уменьшением объемного заряда в подзатворном диэлектрике [2-5]. При длительных временах отжига после этапа роста наблюдается спад плотности латентных ПС [3-5]. Явление связывалось с диффузией молекулярного водорода из прилегающих к МОП структуре областей [2,3], его разложением (крекингом) на положительно заряженных центрах в $\mathrm{SiO}_{2}$ с освобождением ионизованного водорода $\mathrm{H}^{+}$, дрейфом ионов $\mathrm{H}^{+}$ к МФГ $\mathrm{Si}-\mathrm{SiO}_{2}$ и образованию ПС при депассивации $P_{b}$-центров $\left(\mathrm{Si}_{3} \equiv \mathrm{Si \bullet}\right)$ по известной реакции

$$
\equiv \mathrm{SiH}+\mathrm{H}^{+} \rightarrow \equiv \mathrm{Si} \bullet{ }^{+}+\mathrm{H}_{2} .
$$

В работах $[4,5]$ была предложена количественная модель явления, учитывающая кинетику реакций крекинга имеющихся в МОП структуре молекул воды $\mathrm{H}_{2} \mathrm{O}$ на МФГ $\mathrm{Si}-\mathrm{SiO}_{2}$ с образованием нейтральных атомов водорода $\mathrm{H}^{0}$, образования из них молекул водорода $\mathrm{H}_{2}$ и их крекинга на положительно заряженных центрах в оксиде с образованием ионов водорода $\mathrm{H}^{+}$ $(\mathrm{H}-\mathrm{W}$-модель). Модель описывает экспериментальные данные авторов $[4,5]$ по латентному образованию ПС при наборе модельных параметров. Отметим, однако, что в $\mathrm{H}-\mathrm{W}$-модели $[4,5]$ не учитывается транспорт подвижных реагирующих частиц, а временны́е зависимости определяются физически не подтвержденной зависимостью концентрации молекул воды от времени отжига $t$ вида: $\left[\mathrm{H}_{2} \mathrm{O}\right]=k_{7} t \cdot \exp \left(-a t^{n}\right)$, где $k_{7}, a$ и $n-$ эмпирические параметры модели.

В отличие от модели $[4,5]$ полагаем, что исходные ионы водорода $\mathrm{H}^{+}$образуются не в результате крекинга молекул воды $\mathrm{H}_{2} \mathrm{O}$ и молекул водорода $\mathrm{H}_{2}$, а в результате взаимодействия дырок, образующихся при ИО, с водородосодержащими дырочными ловушками. При этом реакция происходит не только в тонком подзатворном оксиде, как в моделях [6,7], описывающих нормальное образование ПС, но и в прилегающем к нему толстом полевом диэлектрике. При решении системы диффузионно-кинетических уравнений, описывающих транспорт подвижных частиц, кинетику их взаимодействия с ловушками и кинетику пассивации и депассивации ПС, учитываем дисперсионный характер транспорта ионов $\mathrm{H}^{+}$к МФГ $\mathrm{Si}-\mathrm{SiO}_{2}$.

\section{2. Описание модели}

Поведение МОП структуры после ИО определяется ее состоянием, сформированным воздействием ИО. В процессе ИО в тонком подзатворном оксиде, а также в прилегающем толстом полевом диэлектрике генерируются электронно-дырочные пары и происходит образование объемного заряда в результате захвата дырок на безводородные дырочные ловушки,

$$
T^{0}+h^{+} \stackrel{k_{1}}{\rightarrow} T^{+},
$$


и их разрядка при захвате электронов,

$$
T^{+}+e^{-\stackrel{k_{2}}{\longrightarrow}} T^{0}
$$

При взаимодействии дырки с водородосодержащими ловушками образуются свободные ионы водорода:

$$
T \mathrm{H}^{0}+h^{+} \stackrel{k_{3}}{\rightarrow} T^{0}+\mathrm{H}^{+}
$$

которые переносятся к МФГ $\mathrm{Si}-\mathrm{SiO}_{2}$, где освобождают от водорода (депассивируют) $P_{b}$-центры, образуя ПС,

$$
P_{b} \mathrm{H}+\mathrm{H}^{+} \underset{k_{s 2}}{\stackrel{k_{s 1}}{\rightleftarrows}} P_{b}^{+}+\mathrm{H}_{2} .
$$

Транспорт ионов $\mathrm{H}^{+}$в аморфной структуре $\mathrm{SiO}_{2}$ имеет дисперсионный характер [6,8], что учитывается посредством реакций захвата ионов водорода на локализованные состояния и освобождения с них:

$$
S_{i}+\mathrm{H}^{+} \underset{k_{r i}}{\stackrel{k_{f i}}{\rightleftarrows}} S_{i} \mathrm{H}^{+}, \quad i=1,2 \ldots k,
$$

где $k_{f i}$ и $k_{r i}$ - константы скоростей прямой реакции захвата и обратной реакции освобождения ионов водорода с локализованных состояний, $k-$ число локализованных состояний.

Система диффузионно-кинетических уравнений непрерывности и уравнения Пуассона, описывающая поведение компонентов реакций (1)-(5) и учитывающая дисперсионный характер транспорта ионов $\mathrm{H}^{+}$при нормальном образовании ПС, имеет вид, приведенный в предыдущей работе автора [7]. Отличие описания латентного накопления ПС заключается в расширении области решения до толщины полевого оксида $x=L$ (полагали $L=10 d$, где $d-$ толщина подзатворного оксида или расстояние от кремниевой подложки до затвора). Другим отличием является расчет скорости образования ПС по кинетике реакции (4). В модели [7] плотность ПС рассчитывалась по интегральному потоку ионов $\mathrm{H}^{+}$на поглощающую границу с кремниевой подложкой, что равносильно очень большой скорости прямой реакции (4). В настоящей модели плотность ПС определяем из уравнения баланса пассивации и депассивации $P_{b}$-центров с учетом скоростей как прямой, так и обратной реакций (4):

$$
\frac{\partial Q_{P_{b}}^{+}}{\partial t}=k_{s 1} Q_{P_{b} \mathrm{H}} C_{\mathrm{H}}^{+}-k_{s 2} Q_{P_{b}}^{+} C_{\mathrm{H}_{2}},
$$

где $Q_{P_{b} \mathrm{H}}$ и $Q_{P_{b}}^{+}$- плотности пассивированных и непассивированных $P_{b}$-центров, $C_{\mathrm{H}}^{+}$и $C_{\mathrm{H}_{2}}-$ концентрации ионов $\mathrm{H}^{+}$и молекулярного водорода $\mathrm{H}_{2}$ на МФГ при $x=0$. Учитываем также возможность крекинга молекул $\mathrm{H}_{2}$ не только на поверхностных $P_{b}$-центрах по обратной реакции (4), но и на объемных положительно заряженных ловушках по реакции, установленной в работе [9]:

$$
\mathrm{H}_{2}+T^{+} \underset{k_{5}}{\stackrel{k_{4}}{\rightleftarrows}} T \mathrm{H}^{0}+\mathrm{H}^{+} .
$$

Соответственно добавляется уравнение диффузии для молекулярного водорода $\mathrm{H}_{2}$ с соответствующими кинетическими членами:

$$
\frac{\partial C_{\mathrm{H}_{2}}}{\partial t}=D_{\mathrm{H}_{2}} \frac{\partial^{2} C_{\mathrm{H}_{2}}}{\partial x^{2}}-k_{4} C_{T}^{+} C_{\mathrm{H}_{2}}+k_{5} C_{T \mathrm{H}}^{0} C_{\mathrm{H}}^{+} .
$$

Как и в [7], полагаем, что исходные концентрации нейтральных безводородных дырочных ловушек распределены вблизи кремниевой подложки по экспоненциальному закону:

$$
C_{T_{0}}^{0}(x)=Q_{T_{0}}^{0} / l_{T} \exp \left(-x / l_{T}\right),
$$

где $Q_{T_{0}}^{0}$ - исходная интегральная концентрация безводородных ловушек, $l_{T}$ - ширина их распределения $\left(l_{T} \sim 5\right.$ нм$)$. Концентрация водородосодержащих дырочных ловушек помимо экспоненциальной имеет однородную составляющую:

$$
C_{T \mathrm{H}_{0}}^{0}(x)=Q_{T \mathrm{H}_{0}}^{0} / L+Q_{T \mathrm{H}_{1}}^{0} / l_{T} \exp \left(-x / l_{T}\right),
$$

где $Q_{T \mathrm{H}_{0}}^{0}$ и $Q_{T \mathrm{H}_{1}}^{0}-$ исходные интегральные концентрации однородно и неоднородно распределенных водородосодержащих дырочных ловушек соответственно.

Уравнения для свободных носителей заряда решались при граничных условиях, соответствующих нулевой концентрации на обеих границах:

$$
p(0, t)=p(L, t)=n(0, t)=n(L, t)=0 .
$$

На границе с подложкой при $x=0$ граничное условие для ионов $\mathrm{H}^{+}$имеет вид

$$
j_{\mathrm{H}}^{+}=\frac{\partial Q_{P_{b}}^{+}}{\partial t},
$$

где $j_{\mathrm{H}}^{+}-$поток ионизованного водорода, $j_{\mathrm{H}}^{+}=-D_{\mathrm{H}}^{+} \frac{\partial C_{\mathrm{H}}^{+}}{\partial x}$ $+\mu_{\mathrm{H}}^{+} C_{\mathrm{H}}^{+} E$.

Граница $x=L$ для ионов $\mathrm{H}^{+}$полагается отражающей:

$$
j_{\mathrm{H}}^{+}(L, t)=0 .
$$

К затвору при $x=d$ относительно подложки приложено напряжение $V_{g}$ :

$$
V(0, t)=0 ; \quad V(d, t)=V_{g} .
$$

Как и в [7], ловушки, определяющие дисперсионный характер транспорта ионов $\mathrm{H}^{+}$, связанные с нарушениями структуры $\alpha-\mathrm{SiO}_{2}$, полагаем распределенными равномерно по толщине диэлектрика:

$$
C_{\mathrm{Si}}^{0}(x, 0)=C_{\mathrm{Si}}^{0}\left(E_{b i}\right), \quad i=1,2 \ldots k,
$$

где $E_{b i}$ - энергия связи иона с ловушкой.

Полагаем, что ионы $\mathrm{H}^{+}$захватываются на ловушки с одинаковой скоростью: $k_{f i}=k_{f}=\sigma_{\mathrm{H}}^{+} v_{d}$, где $\sigma_{\mathrm{H}}^{+}-$ сечение захвата ионов $\mathrm{H}^{+}$на ловушки, $v_{d}$ - дрейфовая скорость, $v_{d}=\mu_{\mathrm{H}}^{+} E$. Освобождение ионов $\mathrm{H}^{+}$с ловушек происходит со скоростью, зависящей от энергии связи иона с ловушкой $E_{b i}, k_{r i}=v \exp \left(-E_{b i} / k_{\mathrm{B}} T\right)$, где $v-$ частотный фактор, соответствующий частоте колебаний 
атомов $\left(v=10^{12} \mathrm{c}^{-1}\right), k_{\mathrm{B}}-$ постоянная Больцмана, $T$ - температура. Распределение плотности локализованных состояний по энергии имеет экспоненциальный вид, характерный для некристаллических и аморфных материалов [8]:

$$
C_{\mathrm{Si}}^{0}\left(E_{b i}\right)=\frac{C_{S}^{0}}{E_{0}} \exp \left(-\frac{E_{b i}}{E_{0}}\right),
$$

где $C_{S}^{0}-$ полная концентрация водородных ловушек, $C_{S}^{0}=\sum_{i=1}^{k} C_{\mathrm{Si}}^{0} ; E_{0}-$ характеристическая энергия, соответствуюшая ширине энергетического распределения ловушек, связанная с дисперсионным параметром $\alpha$ соотношением $\alpha=k_{\mathrm{B}} T / E_{0}, \alpha=0.37$ [7].

Как показано в [7], разрядка положительно заряженных ловушек $T^{+}$осушествляется посредством термоэмиссии с семейства энергетических уровней со скоростью эмиссии

$$
R_{T E j}=A T^{2} \exp \left(-E_{T E j} / k_{\mathrm{B}} T\right)
$$

где $E_{T E j}-$ высота потенциального барьера $j$-й составляющей с энергией термоэмиссии $E_{T E j}=R_{T E 1}$ $+\left(E_{T E k}-E_{T E 1}\right) \cdot j / k, A-$ постоянная Ричардсона $\left(A=120 \mathrm{~A} / \mathrm{cm}^{2} \cdot \mathrm{K}^{2}\right), k$ - количество уровней.

Сдвиг порогового напряжения под действием облучения $\left(\Delta V_{\text {th }}\right)$ складывается из объемной $\left(\Delta V_{\text {ot }}\right)$ и поверхностной $\left(\Delta V_{\text {it }}\right)$ составляющих:

$$
\Delta V_{\mathrm{th}}=\Delta V_{\mathrm{ot}}+\Delta V_{\mathrm{it}}=-\left(Q_{\mathrm{ot}}+Q_{\mathrm{it}}\right) / C_{\mathrm{ox}},
$$

где $C_{\text {ох }}$ удельная емкость подзатворного диэлектрика, $C_{\text {ox }}=\varepsilon \varepsilon_{0} / d, Q_{\mathrm{it}}-$ заряд $\Pi \mathrm{C}, Q_{\mathrm{ot}}-$ эффективный объемный заряд,

$$
Q_{\mathrm{ot}}=\frac{1}{d} \int_{0}^{d} C_{T}^{+}(x)(d-x) d x .
$$

Заряд ПС определяется плотностью ПС, $Q_{\text {it }}=Q_{P_{b}}^{+}$, и находится из уравнения баланса пассивации и депассивации $P_{b}$-центров $(6)$ при начальном значении плотности пассивированных $P_{b}$-центров $Q_{P_{b} \mathrm{H}_{0}}=1 \cdot 10^{13} \mathrm{~cm}^{-2}[10]$.

В модели использовались следующие значения параметров. Константы скоростей реакций с носителями заряда (1)-(3): $k_{1,3}=\sigma_{p 1,3} v_{\text {th }} D_{p} / D_{n} ; k_{2}=\sigma_{n 2} v_{\text {th }}$, где $\sigma_{p}$ и $\sigma_{n}$ - сечения захвата дырок и электронов соответственно, $v_{\text {th }}$ - тепловая скорость электронов, $v_{\text {th }} \approx 10^{7} \mathrm{~cm} / \mathrm{c}$. Учитывалась зависимость от напряженности электрического поля сечений захвата дырок и электронов при значениях сечения захвата в слабых полях дырок $\sigma_{p 01}=2.1 \cdot 10^{-13} \mathrm{~cm}^{2}, \sigma_{p 03}=1.4 \cdot 10^{-14} \mathrm{~cm}^{2}$ и электронов $\sigma_{n 02}=8 \cdot 10^{-14} \mathrm{~cm}^{2}$ (как в $\left.[6,7]\right)$.

Сечение захвата ионов $\mathrm{H}^{+}$на локализованные состояния при дисперсионном транспорте, $\sigma_{\mathrm{H}}^{+}$, зависит от напряженности электрического поля таким же образом, как сечение захвата дырок $\sigma_{p}$ в $\mathrm{SiO}_{2}$ в сильных полях $\sigma_{\mathrm{H}}^{+}=\gamma E^{-0.5}$, где $\gamma=7.5 \cdot 10^{-10} \mathrm{~B}^{0.5} \cdot \mathrm{cm}^{1.5}$ [7].
Полная концентрация локализованных состояний для водородных ловушек $C_{S}^{0}=5 \cdot 10^{22} \mathrm{~cm}^{-3}$. Минимальная и максимальная энергии локализованных состояний определялись из сопоставления с экспериментальными зависимостями, $E_{b 1}=0.76$ эВ,$\quad E_{b k}=1.5$ эВ,$\quad$ при равномерном распределении энергий внутри диапазона: $E_{b i}=E_{b 1}+\left(E_{b k}-E_{b 1}\right) \cdot i / k$. Константы реакций c подвижными молекулами и ионами водорода имеют вид $k_{4, s 1}=4 \pi R_{4, s 1} D_{\mathrm{H}}^{+} \quad$ и $\quad k_{5, s 2}=4 \pi R_{5, s 2} D_{\mathrm{H}_{2}}$, где $\quad R_{4}=R_{s 1}=1 \cdot 10^{-8} \mathrm{~cm}, \quad R_{5}=R_{s 2}=1 \cdot 10^{-10} \mathrm{cM}$, $D_{\mathrm{H}_{2}}=7.2 \cdot 10^{-5} \exp \left(-0.58 / k_{\mathrm{B}} T\right) \mathrm{cm}^{2} / \mathrm{c}[11]$. Коэффициент диффузии ионов водорода в подзатворном оксиде $D_{\mathrm{H}}^{+}=1.0 \cdot \exp \left(-0.73 / k_{\mathrm{B}} T\right) \quad[12]$, а в полевом диэлектрике $D_{\mathrm{H}}^{+}=5 \cdot 10^{-16} \mathrm{~cm}^{2} /$ c. Таким образом, варьируемыми параметрами модели являются исходные интегральные концентрации водородосодержащих, $Q_{T \mathrm{H}_{0}}^{0}$, и безводородных, $Q_{T_{0}}^{0}$, дырочных ловушек, зависящие от технологии выращивания подзатворного и полевого диэлектриков.

\section{3. Результаты расчетов и их обсуждение}

Уравнения для всех компонентов реакций (1)-(8) с начальными условиями (9), (10) и граничными условиями (11)-(14) с учетом (15)-(19) решались численно с использованием неявной и явной разностных схем. Решения сравнивались с экспериментальными зависимостями плотности поверхностных состояний и объемного заряда в МОП транзисторах от суммарного времени ИО и последующего отжига, полученными в работе [3].

После окончания импульса ИО электроны и дырки, имеющие высокие подвижности, покидают подзатворный и полевой диэлектрики, а освобожденные по реакции (3) ионы $\mathrm{H}^{+}$дисперсионно переносятся к кремниевой подложке, образуя ПС по реакции (4). Зависимости поверхностной $\left(1,1^{\prime}\right)$ и объемной $\left(2,2^{\prime}\right)$ составляющих сдвига порогового напряжения от суммарного времени ИО и длительной выдержки показаны на рис. 1. На рисунке точками 1 и 2 приведены экспериментальные данные работы [3], полученные после рентгеновского (10 кэВ) или $\gamma$-облучения (1 МэВ) с дозой 75 крад при мощности дозы 240 крад $\left(\mathrm{SiO}_{2}\right) / \mathrm{c} \mathrm{MOП} p$-структур с подзатворным оксидом толщиной 50 нм. Облучение и отжиг при температуре $T=100^{\circ} \mathrm{C}$ проводились при напряжении на затворе $V_{g}=+6$ В. Расчетные кривые $\left(1^{\prime}, 2^{\prime}\right)$ для процесса ИО (пунктирные линии) и после ИО (сплошные линии) получены при $Q_{T}^{0}=8 \cdot 10^{11} \mathrm{~cm}^{-2}, Q_{T \mathrm{H}_{0}}^{0}=1.2 \cdot 10^{10} \mathrm{~cm}^{-2}$ и $Q_{T \mathrm{H}_{1}}^{0}=8 \cdot 10^{12} \mathrm{~cm}^{-2}$ с учетом дисперсии уровней ловушек для ионов $\mathrm{H}^{+}$от $E_{b 1}=0.76$ эВ до $E_{b k}=1.5$ эВ. Как видно из рисунка, при $t>10^{5}$ с поверхностная составляющая сдвига порогового напряжения $\left(\Delta V_{\mathrm{it}}\right)$ начинает расти вплоть до насыщения при $t>10^{6} \mathrm{c}$. Этап роста $\Delta V_{\text {it }}$ сопровождается ускоренным падением объемной составляющей сдвига порогового напряжения $\left(\Delta V_{\text {ot }}\right)$, 


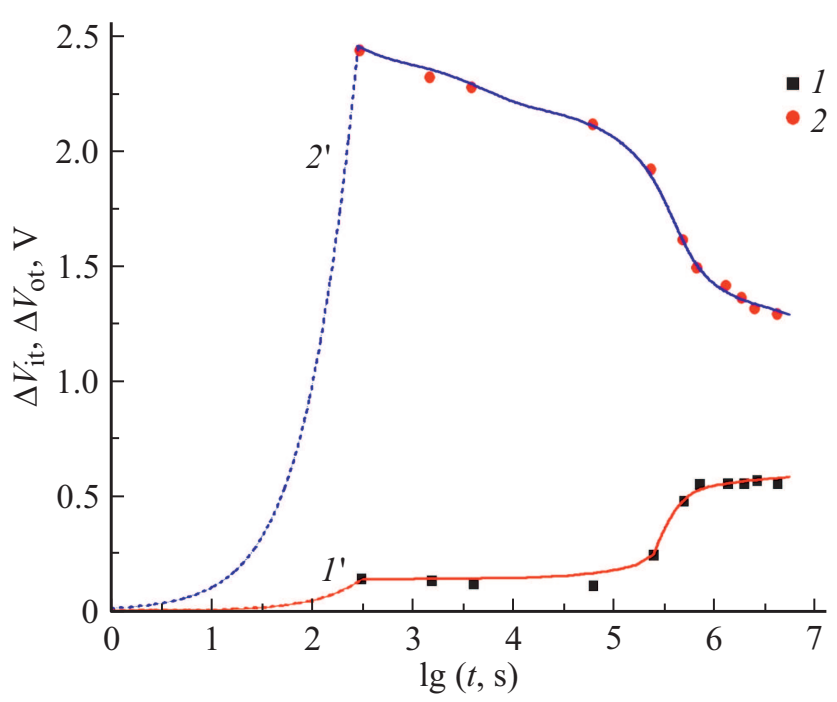

Рис. 1. Кинетика накопления поверхностной, $\Delta V_{\text {it }}\left(1,1^{\prime}\right)$, и объемной, $\Delta V_{\text {ot }}\left(2,2^{\prime}\right)$, составляющих сдвига порогового напряжения: 1,2 - эксперимент [3] (доза 75 крад, $d=50$ нм, $\left.V_{g}=+6 \mathrm{~B}, T=100^{\circ} \mathrm{C}\right) ; 1^{\prime}, 2^{\prime}-$ расчет для ИО (пунктирная линия) и последующего отжига (сплошная линия), $Q_{T}^{0}=8 \cdot 10^{11} \mathrm{~cm}^{-2}, Q_{T \mathrm{H}_{0}}^{0}=1.2 \cdot 10^{10} \mathrm{~cm}^{-2}, Q_{T \mathrm{H}_{1}}^{0}=8 \cdot 10^{12} \mathrm{~cm}^{-2}$.

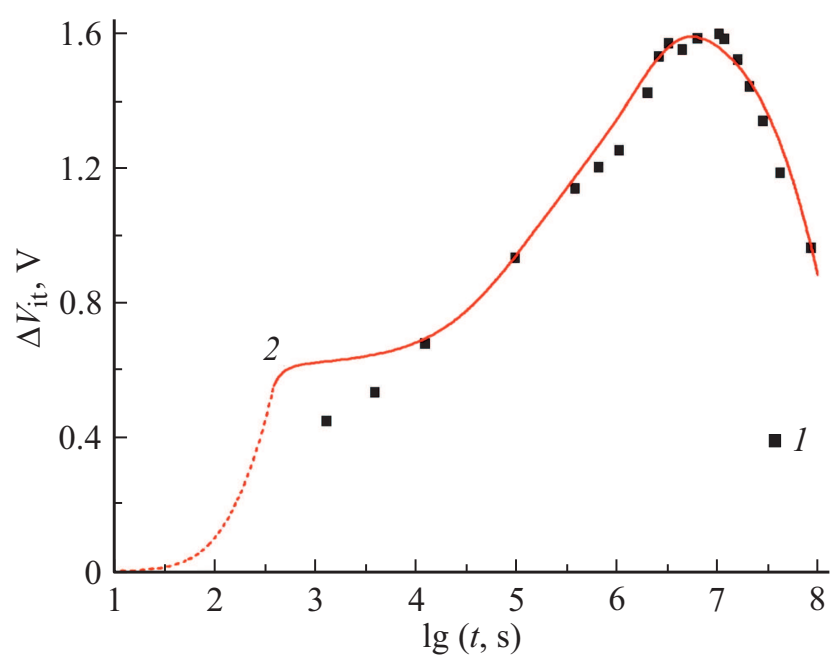

Pис. 2. Кинетика накопления поверхностной составляющей сдвига порогового напряжения $\Delta V_{\mathrm{it}}: 1-$ эксперимент [3] (доза 90 крад, $d=45 \mathrm{Hм}, V_{g}=+6 \mathrm{~B}, T=100^{\circ} \mathrm{C}$ ); $2-$ расчет для ИО (пунктирная линия) и последующего отжига (сплошная линия), $Q_{T}^{0}=2.5 \cdot 10^{13} \mathrm{~cm}^{-2}, Q_{T \mathrm{H}_{1}}^{0}=3.5 \cdot 10^{13} \mathrm{~cm}^{-2}$, $Q_{T \mathrm{H}_{2}}^{0}=8 \cdot 10^{12} \mathrm{~cm}^{-2}$.

которое завершается после выхода $\Delta V_{\text {it }}$ на насыщение. Релаксация объемного заряда происходит путем термоэмиссии с семейства энергетических уровней в диапазоне от $E_{T E 1}=0.7$ эВ до $E_{T E k}=2.0$ эВ (кривая $\left.2^{\prime}\right)$.

Начальный уровень $\Delta V_{i t}$ образуется в процессе ИО вследствие присутствия в приповерхностном слое экспоненциальной составляющей водородосодержащих ловушек с интегральной концентрацией $Q_{T \mathrm{H}_{1}}^{0}$. Согласно модели, последующий рост $\Delta V_{\text {it }}$ связан с приходом ионов $\mathrm{H}^{+}$из области толстого оксида к МФГ $\mathrm{Si}-\mathrm{SiO}_{2}$. При достижении МФГ происходит реакция (4) с депассивацией ПС и образованием молекулярного водорода $\mathrm{H}_{2}$. Последний диффундирует от МФГ в глубь оксида, где приводит к нейтрализации части ловушек $T^{+}$, отвечающих за объемный заряд по реакции (7). Начало роста $\Delta V_{\text {it }}$ определяется толщиной толстого оксида, коэффициентом диффузии ионов $\mathrm{H}^{+}$в толстом оксиде и начальной концентрацией водородосодержащих ловушек в МОП-структуре. Насыщение $\Delta V_{\text {it }}$ связано с уменьшением концентрации положительно заряженных ловушек $T^{+}$в реакции (7). Величина насыщения определяется начальной концентрацией безводородных ловушек $Q_{T_{0}}^{0}$. Соответствующая плато плотность накопленных ПС значительно превышает величину $Q_{T \mathrm{H}_{0}}^{0}$, что связано с появлением добавочных ионов $\mathrm{H}^{+}$при крэкинге молекул $\mathrm{H}_{2}$ по реакции (7). По этой же реакции происходит сопутствующее накоплению ПС падение объемного заряда.

При длительных временах отжига после стадии роста наблюдался спад плотности латентных ПС [3-5]. На рис. 2 точками 1 приведены экспериментальные данные работы [3], полученные после рентгеновского (10 кэВ) или $\gamma$-облучения (1 МэВ) с дозой 90 крад при мощности дозы 240 крад $\left(\mathrm{SiO}_{2}\right) / \mathrm{c} \mathrm{MOП} p$-структур с подзатворным оксидом толщиной 45 нм. Облучение и отжиг проводились при температуре $T=100^{\circ} \mathrm{C}$ и напряжении на затворе $V_{g}=+6 \mathrm{~B}$. Перед ИО МОП структуры подвергались специальному высокотемпературному отжигу $\left(1100^{\circ} \mathrm{C}\right)$ в среде азота. В процессе такого отжига на МФГ с поликремниевым затвором происходит реакция разложения диоксида кремния $\mathrm{SiO}_{2}+\mathrm{Si} \rightarrow 2 \mathrm{SiO}$ [13] с генерацией кислородных вакансий. Последние захватывают водород, содержащийся в значительных количествах на обеих МФГ, с кремнием и поликремнием [14], образуя таким образом дополнительные водородосодержащие дырочные ловушки. Учтем этот вклад в начальное распределение водородосодержащих ловушек в виде экспоненты, спадающей в глубь полевого оксида (при $x \geq d)$ :

$$
C_{T \mathrm{H}_{0}}^{0}(x)=Q_{T \mathrm{H}_{2}}^{0} / l_{T \mathrm{H}} \exp \left[-(x-d) l_{T \mathrm{H}}\right],
$$

где $Q_{T \mathrm{H}_{2}}^{0}-$ исходная интегральная концентрация неоднородно распределенных водородосодержащих дырочных ловушек в полевом диэлектрике, $l_{T \mathrm{H}}$ - ширина распределения $\left(l_{T \mathrm{H}}=0.7 d\right)$. Расчетная кривая 2 в процессе ИО (пунктирная линия) и после ИО (сплошная линия) получены при $Q_{T \mathrm{H}_{1}}^{0}=3.5 \cdot 10^{13} \mathrm{~cm}^{-2}$ и $Q_{T \mathrm{H}_{2}}^{0}=8 \cdot 10^{12} \mathrm{~cm}^{-2}$. Неоднородное распределение водородосодержащих дырочных ловушек в виде (20) обеспечивает монотонный рост ПС уже при относительно небольших временах отжига. Падение ПС при временах $t>10^{7}$ с обусловлено обратной реакцией (4) при превышении на МФГ концентрации образующегося молекулярного водорода над концентрацией поступающего ионизованного водорода. На рис. 1 падение $\Delta V_{\mathrm{it}}(t)$ за наблюдаемое время отжига не происходит вследствие 
более низкой исходной интегральной концентрации водородосодержащих дырочных ловушек.

Отметим, что используемый в модели коэффициент диффузии ионизованного водорода в полевом диэлектрике на несколько порядков меньше литературного значения [12], используемого для подзатворного оксида. Это позволило получить решения при толщине полевого оксида на порядок больше толщины подзатворного оксида. Значительное уменьшение коэффициента диффузии ионизованного водорода в полевом оксиде может означать, что его перенос осуществляется с расстояний, значительно превышающих толщину полевого оксида, например, латерально из прилегающих к МОП-структуре областей.

\section{4. Заключение}

На базе численного решения диффузионно-кинетических уравнений и уравнения Пуассона разработана новая количественная модель латентного накопления ПС после ИО и длительного отжига МОП структур. В основе модели лежит образование ионизованного водорода под действием ИО не только в тонком подзатворном оксиде, но и в прилегающем к нему толстом полевом диэлектрике, и дисперсионный транспорт его к МФГ $\mathrm{SiO}_{2}$ с кремниевой подложкой. Скорость накопления ПС определяется скоростью прямой реакции депассивации $P_{b}$-центров, а скорость уменьшения плотности ПС скоростью обратной реакции их пассивации. Модель позволяет описать экспериментальную кинетику накопления и спада поверхностной составляющей сдвига порогового напряжения $\Delta V_{\text {it }}$ и сопутствующее падение сдвига его объемной составляющей $\Delta V_{\mathrm{ot}}$.

\section{Конфликт интересов}

Автор заявляет, что у него нет конфликта интересов.

\section{Список литературы}

[1] К.И. Таперо, В.Н. Улимов, А.М. Членов. Радиационные эбфекты в кремниевых интегральных схемах космического применения (М., БИНОМ, 2012).

[2] J.R. Schwank, D.M. Fleetwood, M.R. Shaneyfelt, P.S. Winokur. IEEE Electron Dev. Lett., 13 (4), 203 (1992).

[3] J.R. Schwank, D.M. Fleetwood, M.R. Shaneyfelt, P.S. Winokur. C. L. Axness, L.C. Riewe. IEEE Trans. Nucl. Sci., 39 (6), 1953 (1992).

[4] G.S. Ristič, M.M. Pejovič, A.B. Jakšič. J. Appl. Phys., 83 (6), 2994 (1998).

[5] G.S. Ristič, M.M. Pejovič, A.B. Jakšič. J. Appl. Phys., 87 (7), 3468 (2000).

[6] О.В. Александров. ФТП, 54 (2), 189 (2020).

[7] О.В. Александров. ФТП, 55 (2), 152 (2021).

[8] V.I. Arkhipov, A.I. Rudenko. Phil. Mag. B, 45 (2), 189 (1982).

[9] R.E. Stahlbush, A.H. Edwards, D.L. Griscom, B.J. Mrstik. J. Appl. Phys., 73 (2), 658 (1993).

[10] A. Stesmans. Phys. Rev. Lett., 70 (11), 1723 (1993).
[11] J. Fishbein, J.T. Watt, J.D. Plummer. J. Electrochem. Soc., 134 (3), 674 (1987).

[12] S.R. Hofstein. IEEE Trans. Electron Dev., 11 (11), 749 (1967).

[13] H.-E. Sasse, U. König. J. Appl. Phys., 67 (10), 6194 (1990).

[14] Y. Nissan-Cohen. Appl. Surf. Sci., 39, 511 (1989).

Редактор Л.В. Шаронова

\section{Latent accumulation of surface states in MOS structures after ionizing radiation}

\author{
O.V. Aleksandrov
}

St. Petersburg State Electrotechnical University „LETl“, 197376 St. Petersburg, Russia

Abstract A new quantitative model of latent accumulation of surface states in MOS structures after ionizing radiation and prolonged annealing has been developed. The model is based on the formation of $\mathrm{H}^{+}$hydrogen ions under the influence of ionizing radiation not only in a thin gate dielectric, but also in an adjacent thick field dielectric and its subsequent dispersion transport to the interface with a silicon substrate. The density of latent surface states is determined by the balance equation of passivation and depassivation of $P_{b}$-centers on the $\mathrm{SiO}_{2}-\mathrm{Si}$ interface by hydrogen ions. The model allows us to explain the concomitant drop in the volume charge, as well as the decline in the surface states density after the growth is completed, and satisfactorily describes the experimental data. 